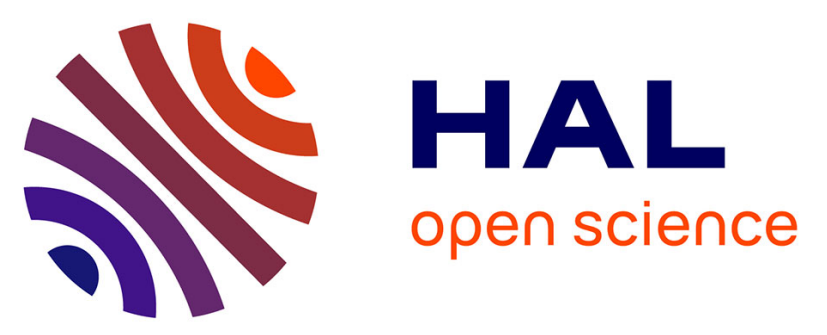

\title{
Insertion of CdSe quantum dots in ZnSe nanowires: Correlation of structural and chemical characterization with photoluminescence
}

Martien den Hertog, Miryam Elouneg-Jamroz, Edith Bellet-Amalric, Samir Bounouar, Catherine Bougerol, Régis André, Yann Genuist, Jean-Philippe Poizat, Kuntheak Kheng, Serge Tatarenko

\section{To cite this version:}

Martien den Hertog, Miryam Elouneg-Jamroz, Edith Bellet-Amalric, Samir Bounouar, Catherine Bougerol, et al.. Insertion of CdSe quantum dots in ZnSe nanowires: Correlation of structural and chemical characterization with photoluminescence. Journal of Applied Physics, 2011, 110, pp.034318. 10.1063/1.3618685 . hal-00721754

\section{HAL Id: hal-00721754 \\ https://hal.science/hal-00721754}

Submitted on 30 Jul 2012

HAL is a multi-disciplinary open access archive for the deposit and dissemination of scientific research documents, whether they are published or not. The documents may come from teaching and research institutions in France or abroad, or from public or private research centers.
L'archive ouverte pluridisciplinaire HAL, est destinée au dépôt et à la diffusion de documents scientifiques de niveau recherche, publiés ou non, émanant des établissements d'enseignement et de recherche français ou étrangers, des laboratoires publics ou privés. 


\title{
Insertion of CdSe quantum dots in ZnSe nanowires: Correlation of structural and chemical characterization with photoluminescence
}

\author{
M. Den Hertog, ${ }^{1, a)}$ M. Elouneg-Jamroz, ${ }^{1}$ E. Bellet-Amalric, ${ }^{2}$ S. Bounouar, ${ }^{1}$ C. Bougerol, ${ }^{1}$ \\ R. André, ${ }^{1}$ Y. Genuist, ${ }^{1}$ J. P. Poizat, ${ }^{1}$ K. Kheng, ${ }^{2}$ and S. Tatarenko ${ }^{1}$ \\ ${ }^{1}$ CEA-CNRS Group "Nanophysique et Semiconducteurs" Institut Néel, CNRS et Université Joseph Fourier, \\ BP 166, Grenoble Cedex 938042 , France \\ ${ }^{2}$ CEA-CNRS Group "Nanophysique et Semiconducteurs" CEA-INAC/UJF-Grenoblel UMR-E, SP2M, 25 rue \\ des Martyrs, Grenoble Cedex 938042 , France
}

(Received 14 April 2011; accepted 22 June 2011; published online 15 August 2011)

\begin{abstract}
ZnSe nanowires with CdSe quantum dot insertions were grown by molecular beam epitaxy using gold as a catalyst. Structural, chemical, and optical properties of the wires and quantum dots were characterized using electron microscopy and photoluminescence spectroscopy. We determined the crystalline structure, the chemical composition, and the size of the quantum dot and established a correlation between quantum dot size and luminescence. As expected, a blueshift of the luminescence was observed for decreasing quantum dot size. The comparison of calculated photoluminescence energy and experimental data seems to indicate that the quantum dots consist of a $\mathrm{Zn}_{\mathrm{x}} \mathrm{Cd}_{1-\mathrm{x}} \mathrm{Se}$ ternary alloy rather than pure CdSe. (C) 2011 American Institute of Physics. [doi:10.1063/1.3618685]
\end{abstract}

\section{INTRODUCTION}

Nanowires (NWs) are considered interesting structures for novel electronic and opto-electronic device functionalities. Different approaches exist to modify the electro-optical properties of these 1D objects. For example, by changing the material composition during the growth, it is possible to change the chemical composition ${ }^{1}$ along the longitudinal or radial directions. This enables the fabrication of well controlled 1D nanoscale heterostructures. ${ }^{2}$ It is also possible, as described in this work, to insert a slice of a low bandgap semiconductor within a high bandgap NW and fabricate a light emitting quantum dot (QD). ${ }^{3}$ The NW geometry offers numerous advantages over the strain-driven Stranski-Krastanow growth ${ }^{4}$ that can also be used to obtain luminescent QDs. Indeed, the absence of a wetting layer yields a better confinement compared to self-assembled QDs. Furthermore, the creation of the QD in a NW is not a strain driven process; therefore, a much larger choice of materials can be considered. This method offers another degree of freedom, as the size and density of the QDs are not imposed by the respective material parameters (the lattice mismatch), but are determined simply by the amount of the low bandgap material supplied to the NW during growth and the initial density and diameter of catalyst particles.

In a previous work, ${ }^{5}$ we observed single photon emission from ZnSe NWs with CdSe QD heterostructures for temperatures as high as $220 \mathrm{~K}$. However, the NWs were randomly oriented on a $\mathrm{Si}(001)$ substrate with its native oxide. No epitaxial relation between the substrate and the NWs was present. The motivation for the present work is to gain control over the NW crystal structure and growth direction using an epitaxial growth that will, in turn, influence the structure

\footnotetext{
a) Author to whom correspondence should be addressed. Electronic mail: martien.den-hertog@grenoble.cnrs.fr.
}

and orientation of the QD insertion with the aim to tune the QD emission properties.

We report on the growth of $\mathrm{ZnSe}$ NWs and on CdSe QD insertions. First, this includes the $\mathrm{ZnSe}$ buffer layers grown on GaAs substrates prior to NW growth and the gold catalyst dewetting. Then, for different substrate conditions and orientations, the crystal structure of the first stages of NW growth and the growth direction of the NWs are described. We evidence that the epitaxial relation between substrate and NW can be used to influence the NW crystal structure and growth direction. Finally, the CdSe QD insertion is analyzed depending on growth conditions and substrate orientation. Complementary transmission electron microscopy (TEM) based techniques are used to study the crystalline structure, chemical composition, and size of NWs and QDs. The results are correlated to optical measurements made on single NWs from the same samples. The experimentally observed photoluminescence (PL) spectra are compared to calculated emission energies as a function of QD size to study possible effects as piezoelectric fields in the CdSe QD or alloying effects with Zn.

\section{EXPERIMENTAL DETAILS}

The NWs are grown by molecular beam epitaxy (MBE), using gold as a catalyst. Samples used in this study are all grown in a solid source MBE system. The source materials for the MBE system are elemental $\mathrm{Zn}(6 \mathrm{~N})$, Se $(6 \mathrm{~N})$, and Cd (6 N). Commercial GaAs (100) and (111)B substrates are first deoxidized: (i) by repeated flashing up to $580^{\circ} \mathrm{C}$ under ultra high vacuum (UHV) ${ }^{6}$ or (ii) by annealing at $640^{\circ} \mathrm{C}$ under As pressure in a connected III-V MBE growth chamber. For this last case, we then grow a $70 \mathrm{~nm} \mathrm{GaAs}$ buffer layer. In order to avoid Ga incorporation in the NWs and to improve the surface quality and the epitaxial relation between the NWs and the substrate, a thin ZnSe buffer layer (about $50 \mathrm{~nm}$ thick) is grown at $280^{\circ} \mathrm{C}$, as described in a 
previous work. ${ }^{7}$ Gold is deposited from an effusion cell on the $\mathrm{ZnSe}$ buffer layer at room temperature in a dedicated metal deposition chamber connected by UHV to the II-VI and III-V growth chambers. The thickness of gold is around $0.1 \mathrm{~nm}$. A dewetting process at $500^{\circ} \mathrm{C}$ was used in order to avoid the creation of nanotrenches ${ }^{8}$ that cause the NWs to grow in directions inclined to the substrate normal in the case of (100) substrates.

$\mathrm{ZnSe} \mathrm{NWs}$ are grown between $300^{\circ} \mathrm{C}$ and $450{ }^{\circ} \mathrm{C}$ with a $\mathrm{Zn}$ :Se beam equivalent pressure (BEP) ratio around 1:4 and pressures in the $10^{-7}$ Torr range. The CdSe insertions are obtained by switching the $\mathrm{Zn}$ flux to Cd flux for 20 to $45 \mathrm{~s}$. The BEP ratio Cd:Se is around 1:3. Typical growth times are 5 to $10 \mathrm{~min}$ for the ZnSe NWs and 20 min for the NWs with a CdSe insertion.

High-resolution TEM (HRTEM) images are realized on a JEOL 4000 EX microscope, operated at $400 \mathrm{kV}$, equipped with a GATAN 2 K CCD camera. A JEOL 3010 microscope operated at $300 \mathrm{kV}$ is used for HRTEM and energy filtered TEM (EFTEM) imaging, equipped with a GATAN $1 \mathrm{~K} \times 1 \mathrm{~K}$ CCD camera. High angle annular dark field scanning transmission electron microscopy (HAADF STEM) images and energy dispersive $\mathrm{x}$ ray spectroscopy (EDX) spectra are obtained on a probe $\mathrm{C}_{\mathrm{s}}$ corrected FEI TITAN TEM at 300 $\mathrm{kV}$ and a Hitachi S 5500 SEM microscope operated at 30 $\mathrm{kV}$. The spectra are fitted and corrected using the Cliff-Lorimer method to obtain a composition profile along the NWs. These different TEM techniques will be used according to the QD size. Indeed, EDX and EFTEM are difficult to use for QD sizes below $4 \mathrm{~nm}$, due to their spatial resolution and relatively long acquisition times that make these techniques sensitive to sample drift.
TEM samples are prepared by a cleaving method, ${ }^{9}$ classical mechanical polishing followed by $\mathrm{Ar}^{+}$ion milling using a GATAN PIPS, or dispersion of the NWs on a holey carbon grid. A thin carbon layer $(5-10 \mathrm{~nm})$ is deposited on the dispersed and cleaved NW samples to reduce movement of the NWs and to protect them from beam damage.

PL spectra of individual NWs are obtained using a standard micro-photoluminescence ( $\mu \mathrm{PL})$ setup operating at a temperature of $\mathrm{T}=4 \mathrm{~K}$ in a helium flow cryostat. The sample is excited either by a continuous wave laser diode emitting at $405 \mathrm{~nm}$ or an argon laser emitting at $488 \mathrm{~nm}$.

\section{RESULTS AND DISCUSSION}

\section{A. Growth of buffer layers}

GaAs and ZnSe buffer layers are deposited on GaAs substrates prior to NW growth to improve the surface quality and ensure good epitaxial growth of ZnSe NWs. The GaAs and ZnSe buffer layers, observed in cross section by HAADF STEM, reveal, in some cases, the presence of particles with a diameter around $10 \mathrm{~nm}$, giving a bright contrast (see Figs. 1(a), 1(b), and 1(c). A gold peak is present in the EDX spectrum obtained on the particles (Fig. 1(e)), while no gold is present if the EDX spectrum is obtained away from a bright particle (Fig. 1(d)). Interestingly, the gold rich particles can be observed in the ZnSe layer (Figs. 1(a) and 1(b)) and even in the GaAs buffer layer, as presented in Fig. 1(c), which means that the particles have migrated through the $\mathrm{ZnSe}$ buffer layer as the gold is deposited after growth of the GaAs and $\mathrm{ZnSe}$ buffer layers, respectively. Gold rich particles are observed in $\mathrm{ZnSe}$ buffer layers grown on GaAs (100) and GaAs (111)B for sample growth temperatures ranging from $350^{\circ} \mathrm{C}$ to $450^{\circ} \mathrm{C}$.
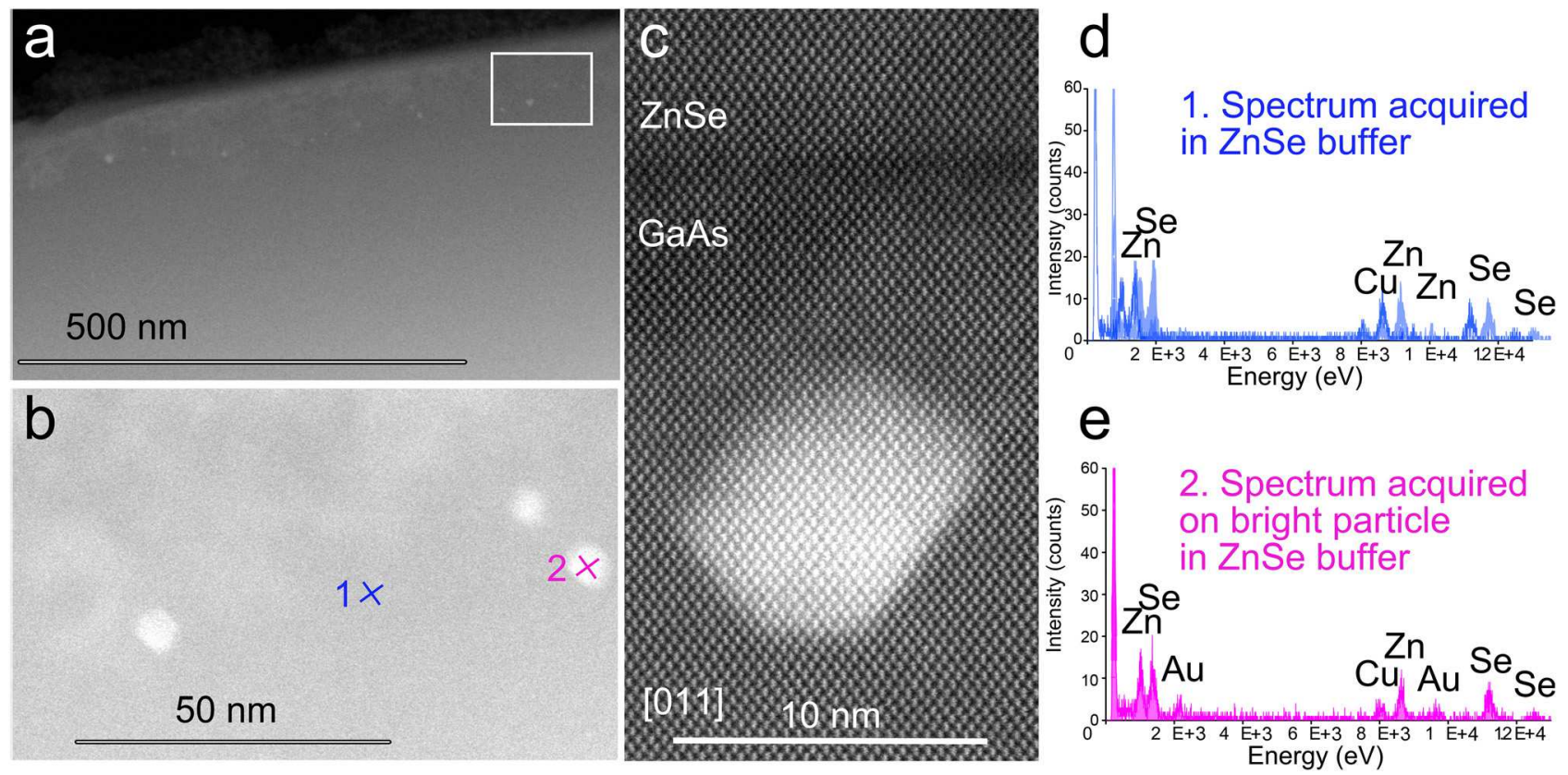

FIG. 1. (Color online) (a) HAADF STEM image of ZnSe on GaAs buffer layers grown on GaAs (100) observed in cross section where bright particles are observed, (b) zoom of the region indicated in (a), (c) HR HAADF STEM image of a buried particle in the GaAs buffer, (d) EDX spectrum obtained in the ZnSe buffer layer at the location marked by 1 in (b), and (e) EDX spectrum obtained on a bright particle at the location marked by 2 in (b). The Cu peak in the EDX spectra is caused by the copper TEM grid. 


\section{B. ZnSe NW growth}

After deposition of buffer layers and dewetting of the gold film, NW growth starts. Both cubic, zinc blende (ZB), and hexagonal wurtzite (WZ) crystal phases can be present in the NWs, depending on the preparation of the starting surface. When present, defects are mostly at the base of the NWs.

NWs grown without a GaAs buffer layer, i.e., on a buffer layer composed only of a $\mathrm{ZnSe}$ layer deposited on a GaAs (100) wafer substrate deoxidized by flashing, grow perpendicular to the substrate or in two distinct inclined directions (see Fig. 2(a)). A HRTEM analysis reveals that, predominantly, the NWs start with the ZB [100] structure with twins and stacking fault defects at the base. This is followed by a transformation of the crystalline structure into the WZ structure along the [0001] direction after 10 to 20 $\mathrm{nm}$ of growth. In Fig. 2(b), the ZB and WZ regions are indicated as well as the twin defect. Although the NW is almost perpendicular to the substrate, the crystallographic structure and orientation are very different from those imposed by the substrate, due to the defect and the change in crystal phase. In other cases, the NWs grow along the ZB $\langle 111\rangle$ or $\langle 112\rangle$ growth directions. The $\langle 112\rangle$ direction generally contains a vertical twin along the length of the NW.
NWs grown using nominally identical growth conditions on substrates with both GaAs and ZnSe buffer layers deposited on GaAs (100) substrates have an improved crystalline quality compared to previous samples (see Fig. 2(c) and 2(d)). The role of the GaAs buffer layer is to reduce the surface roughness, ensuring planar growth of the ZnSe buffer, which appears to reduce the defect density in the NWs. A SEM image of such a sample (Fig. 2(c)) exhibits many vertical NWs. The NWs have the ZB structure and grow in the [100] direction imposed by the substrate, as evidenced by HRTEM (Fig. 2(d)). Some NWs bent under the mechanical action of the glue used for sample preparation. Stacking faults are sometimes observed at the base of NWs; however, these defects change neither the growth direction nor the crystalline structure.

NWs grown on ZnSe with a GaAs buffer layer deposited on GaAs (111)B substrates are nearly all perpendicular to the substrate, as displayed by the SEM image in Fig. 2(e). HRTEM investigations reveal a low crystalline quality with the presence of many defects (Fig. 2(f)). ZB [111] and WZ [0001] oriented regions alternate along the NW, while the NW growth direction remains perpendicular to the substrate.

From the growth of $\mathrm{ZnSe}$ NWs on substrates with different types of preparation, we conclude that the presence of a
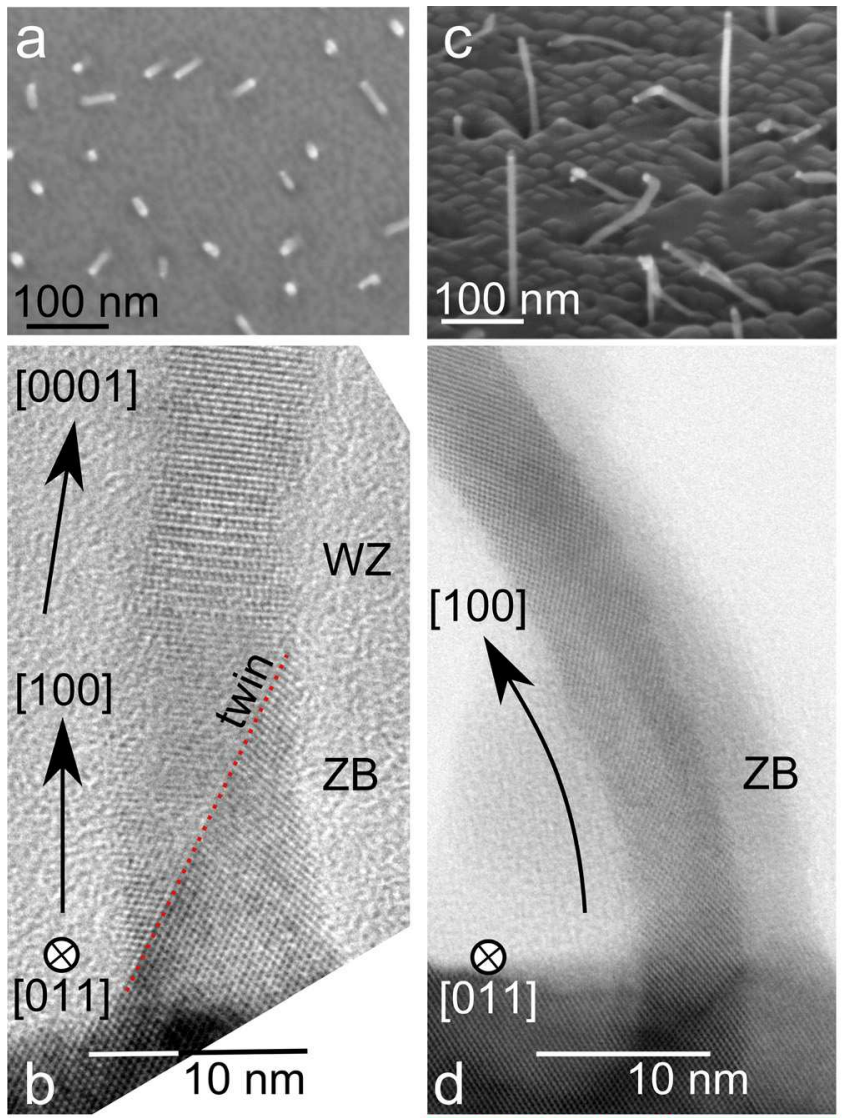

ZnSe buffer

GaAs substrate (100)

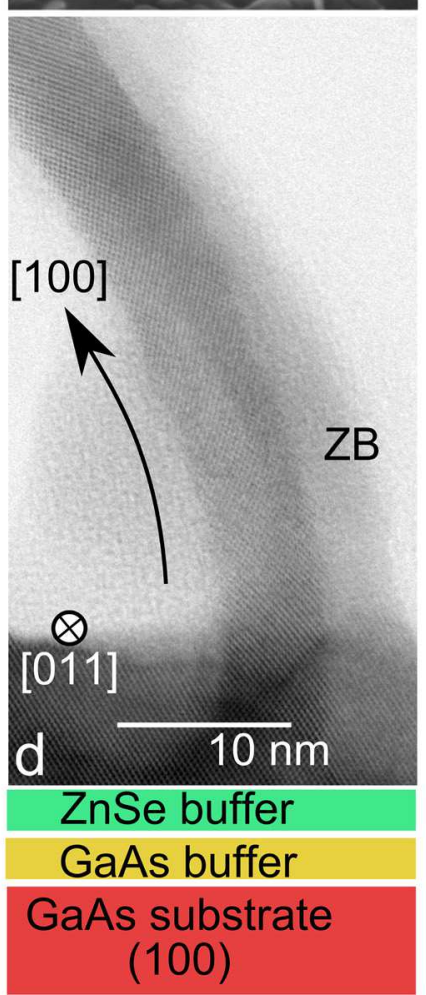

FIG. 2. (Color online) (a) and (b) NWs grown on GaAs (100) with a $\mathrm{ZnSe}$ buffer, as illustrated by the left schematic, (c) and (d) NWs grown on GaAs (100) with a GaAs and $\mathrm{ZnSe}$ buffer, as illustrated by the middle schematic, and (e) and (f) NWs grown on GaAs (111)B with a GaAs and a ZnSe buffer, as illustrated by the right schematic. (a), (c), and (e) SEM images of NWs and (b), (d), and (f) TEM images of a NW from samples (a), (c), and (e), respectively. 


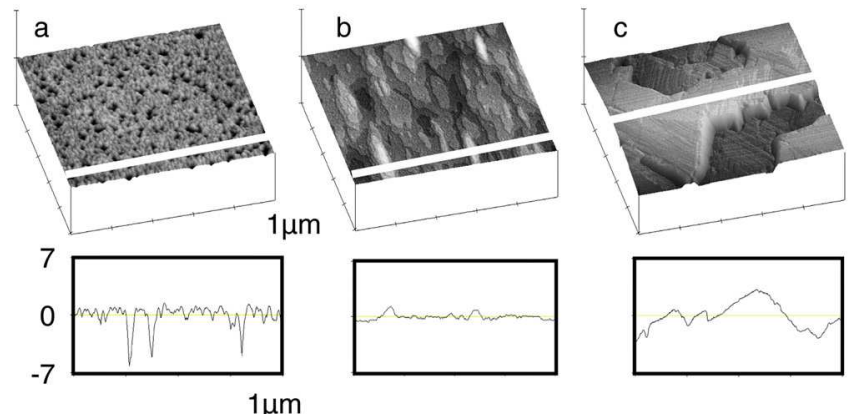

FIG. 3. (Color online) GaAs surface preparation: (a) (100) surface deoxidized by repeated flashing to $580^{\circ} \mathrm{C}$, (b) (100) surface grown by $\mathrm{MBE}$, and (c) (111)B surface grown by MBE. (a), (b), and (c) AFM images at same scale (top) and line profiles (bottom) along the white lines at same scale.

GaAs buffer layer on GaAs (100) substrates enables growth of epitaxial NWs in the ZB [100] direction with a low density of defects. We speculate that, without the GaAs buffer layer, an increased surface roughness can perturb the NW growth, causing twins and stacking fault defects, growth in inclined directions, and eventually a change of crystal phase. Atomic force microscopy (AFM) images illustrate the influence of the preparation on the layer roughness. Indeed, the flash deoxidized GaAs (100) (Fig. 3(a)) has a considerable roughness (root mean square $(\mathrm{rms})=0.95 \mathrm{~nm}$ ) as well as the GaAs buffer layer grown on GaAs (111)B (rms $=0.60 \mathrm{~nm}$, Fig. 3(c)), that might explain the low crystalline quality of NWs. On the contrary, buffer layers of GaAs grown on GaAs (100) (Fig. 3(b)) exhibit the lowest surface roughness $(\mathrm{rms}=0.30 \mathrm{~nm})$.

\section{CdSe QDs growth in ZnSe NWs}

Now that the growth and importance of the buffer preparation for the first stages of NW growth has been described, we turn to the insertion of QDs in the NWs. ZnSe NWs with CdSe QD insertions were studied in detail by HRTEM, HAADF STEM, EFTEM, and EDX measurements, allowing us to characterize the crystalline structure, the chemical composition, and the size of the CdSe QDs. The following six different QD insertions are described in order of decreasing QD size. They exhibit different crystallographic structures and orientations, depending on the growth temperature, substrate orientation, and preparation (see Table I). For each case, $\mu \mathrm{PL}$ measurements were performed on single NWs picked from the same samples.

(IA) Sample IA corresponds to a $30 \mathrm{~s}$ CdSe QD insertion grown at $400^{\circ} \mathrm{C}$. The $\mathrm{ZnSe} \mathrm{NWs}$ were grown following a two step process: ${ }^{10}$ first, a low temperature growth at $300^{\circ} \mathrm{C}$

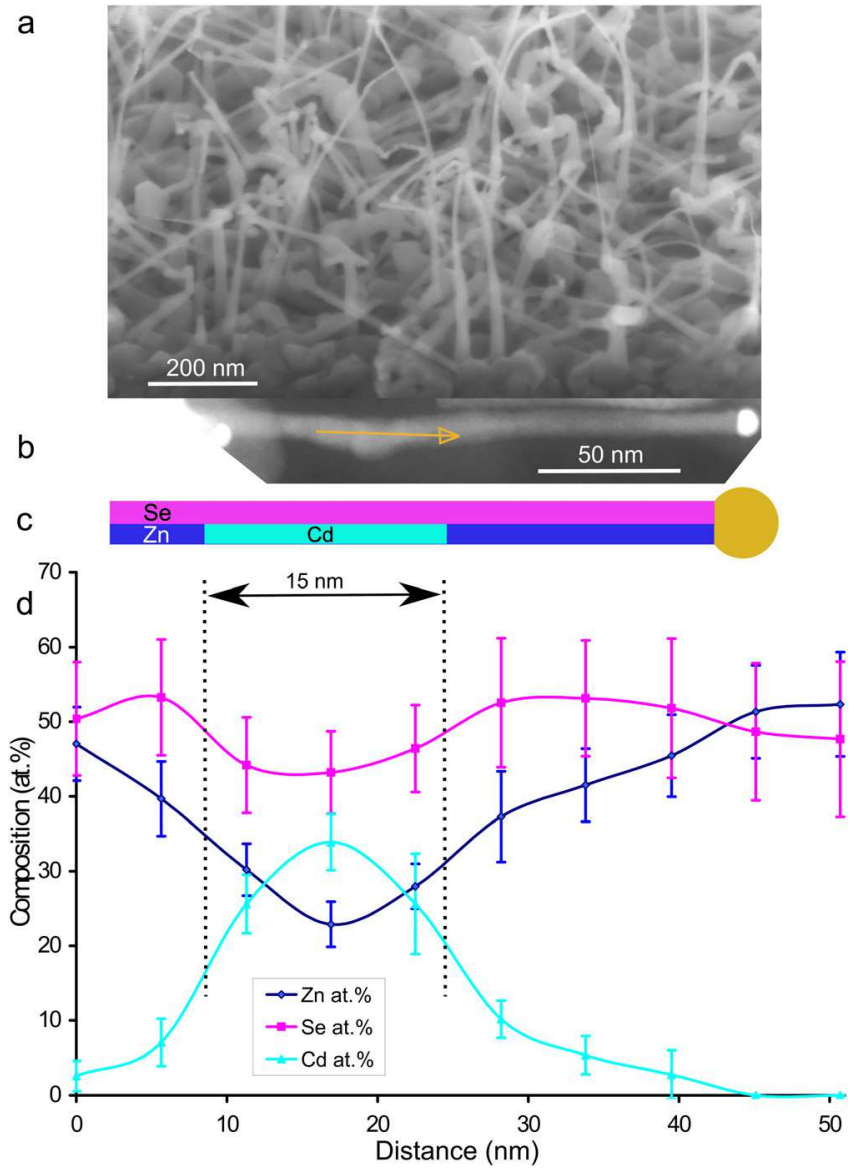

FIG. 4. (Color online) Sample IA: (a) SEM image; (b) SEM image of one NW. The arrow indicates the location of the line scan. (c) Schematic of the NW structure and (d) chemical composition profile along the NW, obtained from treatment of EDX spectra.

was carried out and then a higher temperature was used to grow the part of the NW where the CdSe was inserted. A ZnSe buffer layer deposited on GaAs (100) was used.

A SEM image reveals large tapered and randomly oriented base parts of the NWs, as expected for the low temperature part of the NW (Ref. 10) (Fig. 4(a)). On the contrary, the high temperature growth step gives rise to straight NWs of uniform diameter along the wire. Their diameter and length are rather mono-disperse. We observe a diameter of $10 \pm 5 \mathrm{~nm}$ and a length around $200 \mathrm{~nm}$. The presence of the QD insertion is often marked by the appearance of a thicker part (Fig. 4(b)). The composition of the NW is depicted schematically in Fig. 4(c). An EDX line scan was performed along the NW. Because the NWs are thin, the $\mathrm{x}$ ray count is

TABLE I. Structural and PL parameters of the 6 samples.

\begin{tabular}{|c|c|c|c|c|c|c|c|c|}
\hline QD & $\begin{array}{c}\text { Crystalline } \\
\text { sructure of the NW }\end{array}$ & $\begin{array}{c}\text { Crystalline structure } \\
\text { of the QD }\end{array}$ & QD size $(\mathrm{nm})$ & $\begin{array}{l}\text { QD growth temperature } \\
\text { and duration }\end{array}$ & $\begin{array}{l}\text { Relative Cd pressure } \\
\text { during growth }\end{array}$ & $\begin{array}{c}\text { Interface } \\
\text { width }(\mathrm{nm})\end{array}$ & $\begin{array}{l}\text { PL emission } \\
\text { range (nm) }\end{array}$ & $\begin{array}{l}\text { PL emission } \\
\text { range }(\mathrm{eV})\end{array}$ \\
\hline IA & WZ [0001] & $\mathrm{ZB}[111]$ & $12-18$ & $400^{\circ} \mathrm{C} 30 \mathrm{~s}$ & 1 & $<5 \mathrm{~nm}$ & $530-600$ & $2.34-2.07$ \\
\hline IB & \multicolumn{2}{|l|}{ 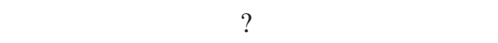 } & $4-5$ & $400{ }^{\circ} \mathrm{C} 30 \mathrm{~s}$ & 1 & $<2 \mathrm{~nm}$ & $530-580$ & $2.34-2.14$ \\
\hline II & \multicolumn{2}{|c|}{ WZ [0001] or ZB [111] } & 3.5 & $400{ }^{\circ} \mathrm{C} 30 \mathrm{~s}$ & $2 / 3$ & $<0.5 \mathrm{~nm}$ & No PL & \\
\hline III & WZ [0001] & WZ [0001] ZB [111] & $2.4-4$ & $410^{\circ} \mathrm{C} 20 \mathrm{~s}$ & 1 & $<0.5 \mathrm{~nm}$ & $520-560$ & $2.39-2.22$ \\
\hline IV & \multicolumn{2}{|c|}{ ZB $[100]$} & 3 & $450^{\circ} \mathrm{C} 45 \mathrm{~s}$ & 1 & $<3 \mathrm{~nm}$ & $490-520$ & $2.53-2.39$ \\
\hline V & WZ [0001] & WZ [0001] ZB [111] & 1.5 & $450{ }^{\circ} \mathrm{C} 30 \mathrm{~s}$ & $1 / 3$ & $<1 \mathrm{~nm}$ & No PL & \\
\hline
\end{tabular}


low, giving a low signal to noise ratio. Also, degradation of the NW under the electron beam is observed. The combination of these factors affects the precision of the composition results (see error bars in Fig. 4(d)). We did not use standards for EDX analysis, but verified that the $\mathrm{Zn}$ and Se composition in the NW were approximately stoichiometric.

A clear $\mathrm{Cd}$ rich region is present at the position of the thicker part of the NW (see Fig. 4(d)). We do not obtain a sharp transition from pure $\mathrm{ZnSe}$ to pure $\mathrm{CdSe}$, but rather a gradual alloying on a length of $\sim 5 \mathrm{~nm}$ around the QD. That alloying also persists throughout the QD. We then define the length of the insertion as the full width half maximum (FWHM) of the Cd peak, which gives a QD of $15 \pm 2 \mathrm{~nm}$ long. The decrease of the Se content in the QD region is unexpected. This could be attributed to Se evaporation at the QD region due to the electron beam, if we consider that the evaporation temperature for $\mathrm{CdSe}$ is much lower than for $\mathrm{ZnSe}$.

To study the crystalline quality of the sample and to obtain chemical maps rather than chemical profiles along a line, we performed HRTEM and EFTEM measurements on a different NW from the same sample (see Fig. 5).

A Zn map obtained using EFTEM (Fig. 5(b)) displays a strong decrease of $\mathrm{Zn}$ signal at the center of the enlargement. In agreement with the previous observation, this droplet shape corresponds to the CdSe QD. The droplet shape of the QD represents an obstacle to line scan measurements of the composition abruptness at the NW interface. It creates a projection of the $\mathrm{ZnSe} \mathrm{NW}$ region over the CdSe QD region in the images and can explain the apparent $\mathrm{Zn}-\mathrm{Cd}$ intermixing on a length of $5 \mathrm{~nm}$ mentioned earlier on EDX measurements. QD lengths ranging from 12 to $18 \mathrm{~nm}( \pm 2 \mathrm{~nm})$ and QD diameters ranging from 6 to $8 \mathrm{~nm}( \pm 2 \mathrm{~nm})$ were observed on this sample on a total of 4 QDs. A Zn rich shell is present around the QD with a thickness of 1-2 $\mathrm{nm}$. This $\mathrm{Zn}$ rich shell is composed of $\mathrm{ZnSe}$ (the diameter of the crystalline core is $10 \mathrm{~nm}$, and the diameter of the $\mathrm{Zn}$ poor region is only 6-8 $\mathrm{nm}$ ) and/or possibly an oxide layer.

The HRTEM images (Figs. 5(a) and 5(c)) exhibit a good crystalline quality all along the wire. A HRTEM zoom of the enlarged region, which corresponds to the CdSe QD region (Fig. 5(c)), reveals that the ZnSe NW presents a WZ structure along the [0001] direction before the QD, while the QD insertion has the ZB structure along the [111] direction. The NW diameter changes from 6 to $10 \mathrm{~nm}$ at the QD insertion, and, after the QD insertion, the crystalline structure remains ZB. Due to the presence of 4 equivalent [111] directions, the modification in the stacking sequence at the inclusion level can explain the kinking of some NWs. Interestingly, bulk ZnSe has the ZB crystal structure, while bulk CdSe has the WZ structure.

The average diameter of the QD is $7 \pm 2 \mathrm{~nm}$ and smaller than its average FWHM length of $15 \pm 3 \mathrm{~nm}$. The presence of the $\mathrm{ZnSe}$ shell could improve the optical properties of the $\mathrm{QD}$, as carriers are less sensitive to possible surface defects (including surface oxidation). A PL spectrum obtained from several NWs (Fig. 5(d)) exhibits a sharp peak at $535 \mathrm{~nm}$ and several smaller peaks. In general, sharp PL peaks with a linewidth around $1.1 \mathrm{~nm}$ are observed on this NW sample in the $530-600 \mathrm{~nm}$ wavelength range.

(IB) Sample IB corresponds to a $30 \mathrm{~s}$ CdSe QD insertion grown at $400^{\circ} \mathrm{C}$. This $\mathrm{NW}$ sample was grown under similar

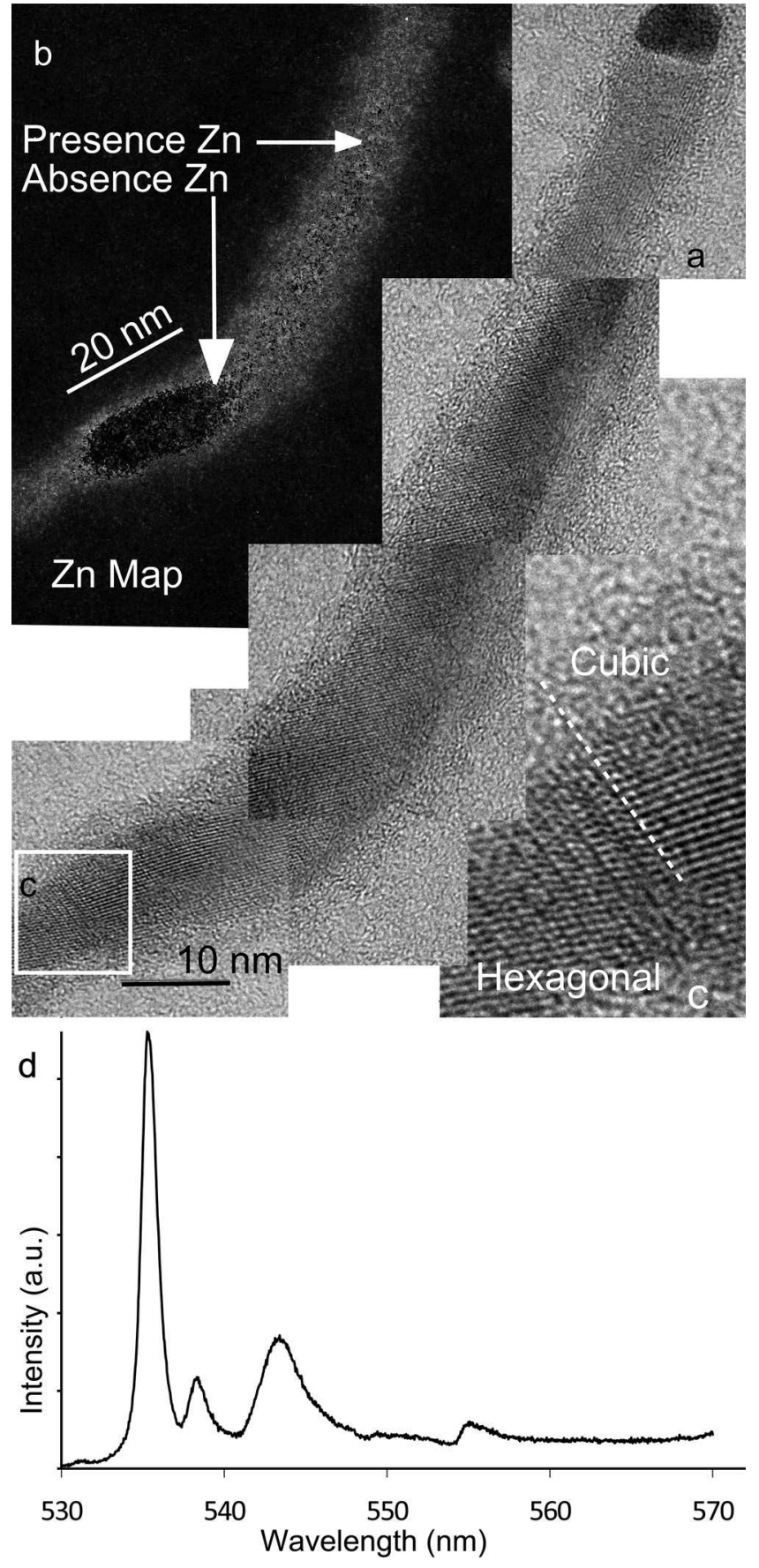

FIG. 5. Sample IA: (a) HRTEM images of a NW, (b) Zn map obtained using EFTEM (using the Zn L edge at $1020 \mathrm{eV}$ ) on the same NW, (c) zoom of the region marked in (a), and (d) PL spectrum of several NWs from the same sample.

growth conditions as sample IA. EFTEM images (not shown) indicate that the QD length is $4.5 \pm 1 \mathrm{~nm}$ and the diameter around $6 \mathrm{~nm}$, as observed on one single NW. The ZnSe NW diameter changes from 10 to $12 \mathrm{~nm}$ at the CdSe QD insertion, and a $\mathrm{Zn}$ rich shell is present around the QD. In general, sharp PL peaks with a linewidth around $0.8 \mathrm{~nm}$ were observed on this NW sample in the 530-580 nm wavelength range (not shown).

(II) Sample II corresponds also to a $30 \mathrm{~s}$ CdSe QD insertion grown at $400^{\circ} \mathrm{C}$, but on a GaAs (111)B substrate with epitaxial $\mathrm{ZnSe}$ and GaAs buffer layers. Only one growth step 
was used. The Cd flow used is $2 / 3$ of the flow used for samples IA and IB. The NWs follow the direction imposed by the substrate, as can be observed on the SEM image of Fig. 6(a). To detect the QDs, we have performed HAADF STEM experiments. As $\mathrm{Cd}$ atoms $(\mathrm{Z}=48)$ are heavier than $\mathrm{Zn}$ and Se atoms, a bright contrast marks the QD insertion (Fig. 6(b)). Clearly, both NWs that grow side by side contain a QD insertion of the same size and at the same distance from the gold particle. A zoom of the region marked in Fig. 6(b) is presented in Fig. 6(d). Only the crystal planes along the growth direction are visible in this experiment. Therefore, it is not possible to determine whether the crystal phase of the NW and the QD are (0001) WZ or (111) ZB. We averaged the interplanar
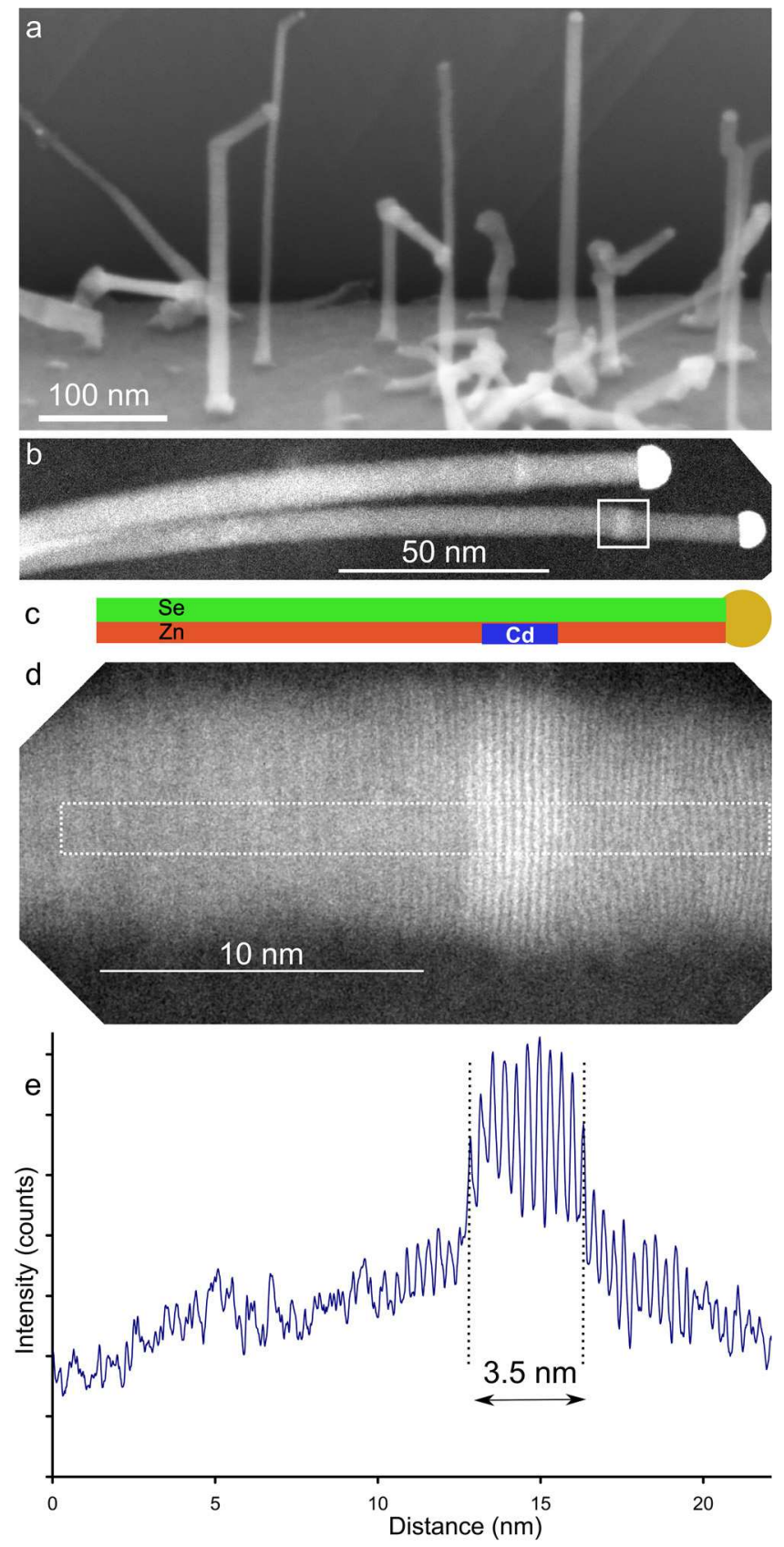

FIG. 6. (Color online) Sample II: (a) SEM image of the NWs, (b) HAADF STEM image of two NWs side by side, (c) schematic illustrating the NW composition, (d) zoom of the region marked in (b), and (e) intensity profile obtained along the dotted rectangle in (d). distance over 10 planes in the $\mathrm{ZnSe}$ and CdSe regions, respectively, using the $\mathrm{ZnSe}$ region as reference (the $\mathrm{ZnSe} \mathrm{ZB} \mathrm{d}_{111}$ spacing is equal to the $\mathrm{WZ} \mathrm{d}_{0002}$ spacing and is $\left.0.327 \mathrm{~nm}\right) .{ }^{37} \mathrm{~A}$ change in lattice parameter from $\mathrm{d}=0.327 \pm 0.1 \mathrm{~nm}$ in the $\mathrm{NW}$ to $\mathrm{d}=0.36 \pm 0.1 \mathrm{~nm}$ in the QD is present in the region of the bright contrast. This is consistent with the expected change in lattice parameter going from $\mathrm{ZnSe}$ to relaxed CdSe. From the STEM intensity profile made along the NW (Fig. 6(e)), we estimate the FWHM size of the QD region with bright contrast to be $3.5 \pm 0.5 \mathrm{~nm}$ (with a very low size dispersion from wire to wire). Almost no Cd-Zn inter-diffusion is present as the intensity drops rapidly at the edges of the brighter region. The linear rise of the HAADF signal in the $\mathrm{ZnSe} \mathrm{NW}$ could be due to small thickness changes, as also observed in sample IA. Indeed, the QD diameter is $9 \mathrm{~nm}$ and the NW diameter before and after the QD is $8 \mathrm{~nm}$ (such a change was observed in 4 out of 5 observed QDs on this sample). It should be noted that no luminescence was observed on this sample. This is attributed to the lower quality of the buffer (see Fig. 2(f)) that might quench the luminescence.

(III) Sample III corresponds to a $20 \mathrm{~s} \mathrm{CdSe} \mathrm{QD} \mathrm{insertion}$ grown at $410^{\circ} \mathrm{C}$ on $\mathrm{ZnSe}$ with a GaAs buffer layer deposited on GaAs (100). The QD length is between 2.4 and $4 \mathrm{~nm}$, as observed in five QDs, and the wire diameter increases from 10 to $11 \mathrm{~nm}$ at the QD position (not shown). The NWs grow in the WZ [0001] direction with defects. Luminescence was observed in the 520-560 $\mathrm{nm}$ range with linewidths around $0.2 \mathrm{~nm}$. Further details of this sample will be described elsewhere.

(IV) Sample IV corresponds to a $45 \mathrm{~s} \mathrm{CdSe} \mathrm{QD} \mathrm{insertion}$ grown at $450^{\circ} \mathrm{C}$ on $\mathrm{ZnSe}$ with a GaAs buffer layer deposited on GaAs (100). This sample contains a first low temperature growth step. Most NWs are vertical, as revealed on the SEM image of Fig. 7(a). The ZB structure and the [100] growth direction of the ZnSe NWs are also confirmed by HAADF STEM analysis, shown on Fig. 7(c). The QD insertion with the same crystal structure and orientation is marked by a brighter contrast. The size of the QD, measured from the intensity profile FWHM made along the NW (Fig. 7(d)), is $3 \pm 0.5 \mathrm{~nm}$, shorter than in previous samples, although the growth time of the QD insertion is 1.5 times longer; but this difference can be explained by the increase in the growth temperature. No thickness change is observed on the NW-QD interface in this sample. The NW diameter is $10 \mathrm{~nm}$. However, in this sample, some $\mathrm{Zn}-\mathrm{Cd}$ intermixing seems to be present at the interface between NW and QD on a length of $3 \mathrm{~nm}$.

It should be noted that a QD insertion along the [100] direction has the advantage that no change of crystal phase or growth direction is present. A PL spectrum obtained on dispersed NWs is presented in Fig. 7(e). Many sharp PL peaks with a linewidth between 0.3 and $0.4 \mathrm{~nm}$ can be observed. The narrow linewidth is an indication of a reduced number of charge traps, which cause spectral diffusion. ${ }^{11}$ The four peaks observed at $493,495,501$, and $503 \mathrm{~nm}$ can be attributed to two sets of exciton (X) - biexciton (XX) emissions from QDs in two separate NWs, as confirmed by measuring the PL intensity as a function of excitation laser power (not shown), which are respectively linear and quadratic, and by cross-correlation experiments. ${ }^{12}$ PL peaks observed on this NW sample are in the $490-520 \mathrm{~nm}$ wavelength range. 

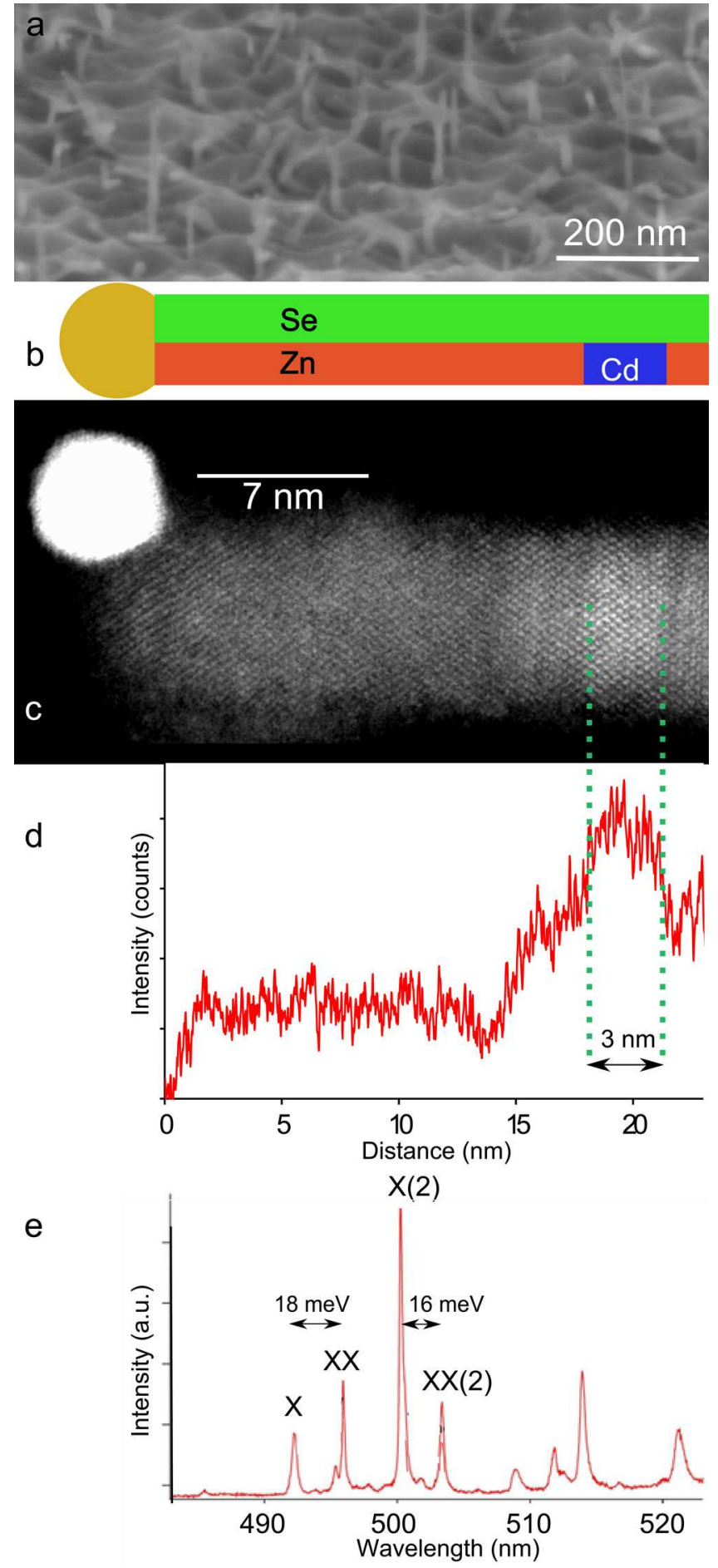

FIG. 7. (Color online) Sample IV: (a) SEM image of the NWs, (b) schematic illustrating the NW composition, (c) HAADF STEM image of one NW, (d) intensity profile made along the NW from (c), and (e) PL spectrum obtained on two NWs from the same sample.

(V) Sample V corresponds to a 30 s CdSe QD insertion grown at $450^{\circ} \mathrm{C}$. The $\mathrm{Cd}$ flow was $1 / 3$ of the flow used for samples I. As discussed previously, NWs grown on a $\mathrm{ZnSe}$ [100] buffer layer on flashed GaAs (100) tend to grow in the WZ [0001] direction with a ZB [111] QD insertion (similar to the NW and QD in Fig. 5). Structural results from this sample were previously presented. ${ }^{8}$ Geometrical phase analysis (GPA) was used on HRTEM images of NWs from this sample to map the interplanar spacing along the wire axis. Since the CdSe (111) interplanar spacing is larger than the ZnSe interplanar spacing, this technique allowed us to obtain the size of the QD and its localization along the NW. Also, the crystallographic structure of the QD is visible in the HRTEM image. The same structural change from $W Z$ to $Z B$, as already observed on sample IA, was reported near the QD region. The QD size was estimated at $\sim 1.5 \mathrm{~nm}$. As in the previous sample, no diameter variation is present at the QD insertion. No PL was observed on this sample.

\section{Optical measurements}

The main purpose of this section is to correlate optical and structural properties of the QDs. Nevertheless, it should be noted that TEM and $\mu \mathrm{PL}$ experiments are not performed on the same unique NW, but on similar neighboring NWs from the same sample. Small wire to wire differences in QD size are therefore possible, as evidenced by the Gaussian broadening of the PL emission wavelength for a NW ensemble over a few tens of nanometers.

A summary of the different types of QD insertions is presented in Table I together with the range of wavelengths where PL was observed. As expected for semiconductor QDs, the confinement energy increases when decreasing the QD size. Indeed, this tendency is confirmed by the optical spectroscopy results represented graphically in Fig. 8, the PL shift being important for QD sizes below $6 \mathrm{~nm}$.

Several possible factors should be considered to explain the observed PL shift:

(i) the confinement associated to the QD size

(ii) the piezoelectric effects along a polar axis due to strain or the spontaneous polarization at the WZ-ZB interface. This effect can be present in insertions (IA) and (III). On the contrary, no piezoelectric or polarization effects could be present in sample IV with growth in the ZB [100] direction. a Cd-Zn alloying.

The calculated curves shown on Fig. 8 are based on electron and hole confinement in a square quantum well (QW) of variable width (associated to the QD length along the NW axis). The barrier bandgap was taken as $2800 \mathrm{meV}$ (440 nm, corresponding to $\mathrm{ZB} \mathrm{ZnSe}$ at $4 \mathrm{~K}$ ) and the QW bandgap as $1750 \mathrm{meV}(673 \mathrm{~nm}$, corresponding to $\mathrm{ZB} \mathrm{CdSe}$ at $4 \mathrm{~K}$ (Ref. 13)). The slightly larger bandgap of WZ ZnSe (present in samples IA and III, that is $2870 \mathrm{meV}$ or $432 \mathrm{~nm}$ ), was not included in the calculation, since this would hardly affect the confinement energy. For pure $\mathrm{CdSe}$, we consider the effect of an electric field of $100 \mathrm{meV} \mathrm{nm}^{-1}$ (triangular well) that could derive from piezoelectric effects. This value of the electrical field is among the lowest values reported for 2D polar QWs, based on similar II-VI materials. ${ }^{14}$ To account for a possible alloying effect, calculations were also performed with a $\mathrm{Zn}$ concentration of 20 to $50 \%$ of element-II (see Fig. 8). For these calculations, the bandgap in the QD material is varied linearly with composition, neglecting a possible slight bowing. ${ }^{15}$

The general trend of the data points clearly shows that there is no polarization field in the studied samples. 


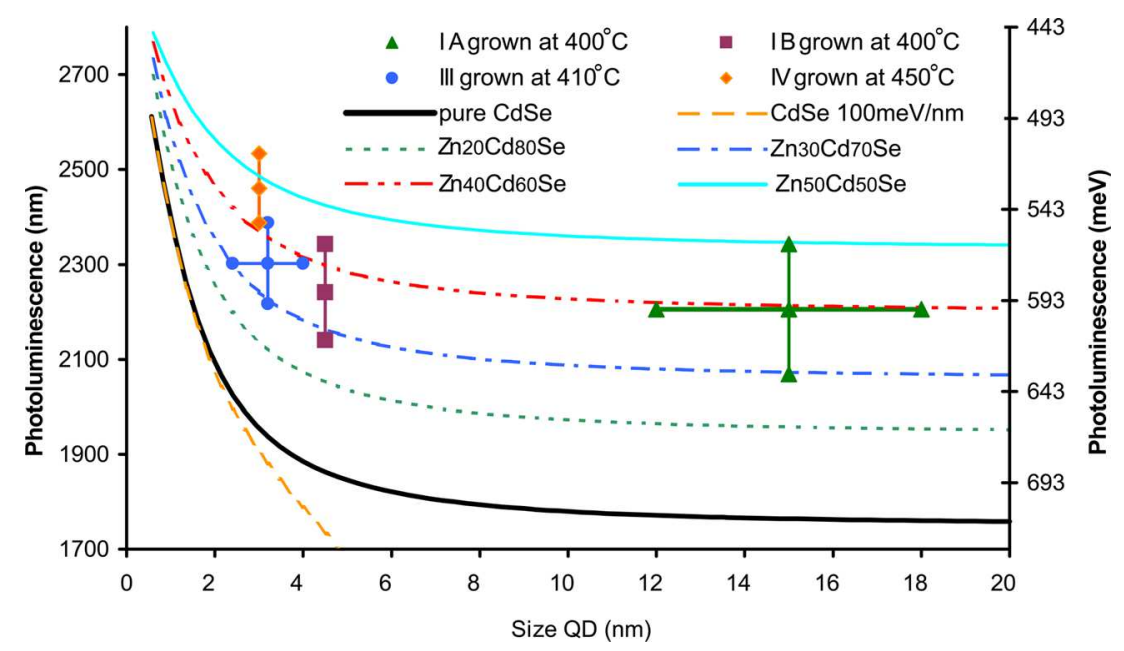

FIG. 8. (Color online) Photoluminescence energy vs QD size for sample IA ( $\mathbf{\Delta}$ ) grown at $400^{\circ} \mathrm{C}$, IB (ם) grown at $400^{\circ} \mathrm{C}$, III (•) grown at $410^{\circ} \mathrm{C}$, and IV (•) grown at $450^{\circ} \mathrm{C}$. For comparison, a set of calculated curves is provided for different assumptions for the QW: (-) pure CdSe, (- -$)$ pure CdSe under an electric field of $100 \mathrm{meV} / \mathrm{nm}$, (-) $\mathrm{Zn}_{50} \mathrm{Cd}_{50} \mathrm{Se}$, (一 • ) $\mathrm{Zn}_{40} \mathrm{Cd}_{60} \mathrm{Se}$, (- .) $\mathrm{Zn}_{30} \mathrm{Cd}_{70} \mathrm{Se}$, and (- - -) $\mathrm{Zn}_{20} \mathrm{Cd}_{80} \mathrm{Se}$. Size error bars are based on the distribution of values obtained by TEM observations, and energy errors bars are based on the PL emission range of a given sample.
However, a strong global shift on the order of $300 \mathrm{meV}$ is observed between data points and calculations. This shift is much larger than the consequences of the simplifying hypotheses of the model. The experimental results are well accounted for by calculation without an electric field but some alloying. It can be observed that the experimental PL values of QDs grown at $400^{\circ} \mathrm{C}$ are between the calculations for $\mathrm{Zn}_{0.3} \mathrm{Cd}_{0.7} \mathrm{Se}$ and $\mathrm{Zn}_{0.4} \mathrm{Cd}_{0.6} \mathrm{Se}$, while the experimental $\mathrm{PL}$ values of the $\mathrm{QD}$ grown at $450^{\circ} \mathrm{C}$ are close to the calculation for $\mathrm{Zn}_{0.5} \mathrm{Cd}_{0.5} \mathrm{Se}$. It seems therefore plausible to assert that the QD is a ternary alloy.

The radial directions of confinement due to the QD and NW diameter were neglected in the calculation. Based on the confinement of an electron-hole pair in a cylinder with infinite barrier ${ }^{16}$ for diameters of typically $10 \mathrm{~nm}$ (the NW diameter), it can be estimated that the radial contribution to the confinement energy would be, at most, a few tens of $\mathrm{meV}$, far below the experimental error bars or the $\sim 300$ meV shift observed between experimental data points and the calculated curve for pure CdSe QDs. The comparison between calculation and experimental points on Fig. 8 provides a clear evidence of a large presence of $\mathrm{Zn}$ in the QDs of more than $30 \%$, even if the simple model we used cannot provide a more accurate value of the $\mathrm{Zn}$ content.

If the QD is a ternary alloy, as suggested from the comparison of experiment and calculation, this should be visible in the TEM analysis. In the following, we look back at the TEM evidence to see if this could be observed and understood, and we speculate on a possible difference in alloy composition depending on the growth temperature.

Indeed, the EDX analysis of sample IA (Fig. 4) shows that the $\mathrm{Zn}$ concentration decreases only to approximately $40 \%$ of element-II. Concerning EFTEM data, it is not possible to exclude the presence of a low Zn quantity in the QD region, as data are relatively noisy. The HAADF STEM measurements (Figs. 6 and 7) exhibit a chemical contrast, but the intensity is not quantitative. Therefore, it is difficult to exclude the presence of low $\mathrm{Zn}$ quantities in the QD region.

For sample V, using the geometrical phase analysis, ${ }^{8}$ an interplanar spacing below the value expected for pure CdSe is found in the QD region, indeed indicating an alloying effect.
The presence of $\mathrm{Zn}$ in the $\mathrm{CdSe}$ region could be caused by the reservoir effect of $\mathrm{Zn}$ and $\mathrm{Cd}$ in the catalyst particle. The composition of the gold catalyst particle after growth was studied by EDX analysis, and no or very low concentrations of $\mathrm{Zn}$ or Cd were observed (not significant given the error bars). As this analysis is performed after growth, it cannot be excluded that the $\mathrm{Zn}$ or $\mathrm{Cd}$ concentration in the catalyst particle is more substantial during growth. Since a diameter increase was observed systematically at the QD insertion for growth temperatures of $400{ }^{\circ} \mathrm{C}$, incorporation of $\mathrm{Cd}$ in the catalyst particle is not impossible. Furthermore, the reservoir effect could be enhanced by the fact that the growth temperature of the QD region is above the CdSe evaporation temperature. Indeed, pure CdSe NWs cannot grow at temperatures above $350^{\circ} \mathrm{C}$ in MBE. A small $\mathrm{Zn}$ concentration can protect the QD regions from evaporation. In other words: the pure $\mathrm{CdSe}$ compound is not thermodynamically stable above $350^{\circ} \mathrm{C}$, and only a ternary alloy can grow at higher temperatures. This implies that increasing $\mathrm{Zn}$ concentrations are expected in the QD for increasing growth temperatures, as observed in Fig. 8. Furthermore, it was observed that, for a similar QD growth time, smaller QDs are obtained at $450{ }^{\circ} \mathrm{C}$ (samples IV and V) than at $400^{\circ} \mathrm{C}$ (samples I, II, and III at $410^{\circ} \mathrm{C}$ ), which is attributed to the reduced sticking of $\mathrm{Cd}$ at higher temperatures caused by an increased evaporation of $\mathrm{Cd}$.

\section{SUMMARY AND CONCLUSION}

We have shown epitaxial growth of ZnSe NWs with luminescent CdSe QD insertions. First of all, the presence of a $\mathrm{ZnSe}$ buffer is crucial to obtain an epitaxial relation between the substrate and the NWs. The surface preparation, defined by the deoxidation method of the GaAs substrate, the presence of a GaAs buffer, and the orientation of the substrate (100) or (111)B, influence the crystallographic quality, growth direction, and crystallographic structure of the NW and QD insertion. The QD can be inserted both in a polar [111] direction, where piezoelectric effects can play a role, and in a non-polar [100] direction. Luminescence was observed from these QDs, and the emission wavelength can be correlated with the size of the QD insertion. Comparison 
of the experimental data with calculated PL energies for a square QW indicate that no influence of polarization on the PL energy is present and that the QD is in fact a $\mathrm{Zn}_{\mathrm{x}} \mathrm{Cd}_{1-\mathrm{x}} \mathrm{Se}$ ternary alloy with increasing $\mathrm{Zn}$ concentration for higher QD growth temperature.

\section{ACKNOWLEDGMENTS}

This work is supported by the French National Research Agency (ANR) through the Nanoscience and Nanotechnology Program (Project BONAFO nANR-08-NANO-031-01), who provided a research fellowship for M.dH. M.E.J. acknowledges financial support from the Nanosciences Foundation "Nanosciences, aux limites de la nanoélectronique" (RTRA). Transmission electron microscopy was performed at PFNC-Minatec Grenoble.

${ }^{1}$ M. S. Gudiksen, L. Lauhon, J. Wang, D. C. Smith, and C. M. Lieber, Nature 415, 617 (2002)

${ }^{2}$ M. T. Björk, B. J. Ohlsson, T. Sass, A. I. Persson, C. Thelander, M. H. Magnusson, K. Deppert, L. R. Wallenberg, and L. Samuelson, Nano Lett. 2, 87 (2002); Appl. Phys. Lett. 80, 1058 (2002).

${ }^{3}$ M. T. Borgström, V. Zwiller, E. Muller, and A. Imamoglu, Nano Lett. 5, 1439 (2005).
${ }^{4}$ E. Bauer, Z. Kristallogr. 110(1-6), 372 (1958).

${ }^{5}$ A. Tribu, G. Sallen, T. Aichele, R Andre, J. Ph. Poizat, C. Bougerol, S. Tatarenko, and K. Kheng, Nano Lett. 8, 4326 (2008).

${ }^{6}$ I. C. Robin, R. André, H. Mariette, S. Tatarenko, L. S. Dang, J. M. Gérard, and E. Bellet-Amalric, Physica E (Amsterdam) 26, 119 (2005).

${ }^{7}$ I. C. Robin, T. Aichele, C. Tatarenko, E. Bellet-Amalric, B. Van Daele, and G. Van Tendeloo, Nanotechnology 18, 265701 (2007).

${ }^{8}$ E. Bellet-Amalric, M. Elouneg-Jamroz, C. Bougerol, M. Den Hertog, Y. Genuist, S. Bounouar, J. P. Poizat, K. Kheng, R. André, and S. Tatarenko, Phys. Status Solidi C 7, 1527 (2010).

${ }^{9}$ M. den Hertog, H. Schmid, D. Cooper, J. L. Rouviere, M. T. Björk, H. Riel, P. Rivallin, S. Karg, and W. Riess, Nano Lett. 9, 3837 (2009).

${ }^{10}$ T. Aichele, A. Tribu, C. Bougerol, K. Kheng, R. André, and S. Tatarenko, Appl. Phys. Lett. 93, 143106 (2008).

${ }^{11}$ G. Sallen, A. Tribu, T. Aichele, R. André, L. Besombes, C. Bougerol, M. Richard, S. Tatarenko, K. Kheng, and J. Ph. Poizat, Nature Photon. 4, 696-699 (2010)

${ }^{12}$ G. Sallen, A. Tribu, T. Aichele, R. André, L. Besombes, C. Bougerol, S. Tatarenko, K. Kheng, and J. Ph. Poizat, Phys. Rev. B 80, 085310 (2009)

${ }^{13}$ O. Zakharov, A. Rubio, X. Blase, M. L. Cohen, and S. G. Louie, Phys. Rev. B 50, 10780 (1994).

${ }^{14}$ R. André, C. Deshayes, J. Cibert, L. S. Dang, S. Tatarenko, and K. Saminadayar, Phys. Rev. B 42, 11392 (1990).

${ }^{15}$ N. Samarth, H. Luo, J. K. Furdyna, R. G. Alonso, Y. R. Lee, A. K. Ramdas, S. B. Qadri, and N. Otsuka, Appl. Phys. Lett. 56, 1163 (1990).

${ }^{16}$ K. L. Teo, S. H. Kwok, and P. Y. Yu, Phys. Rev. B 62, 1584 (2000).

${ }^{17}$ Y. S. Park and F. L. Chan, J. Appl. Phys. 36, 800 (1965). 